\title{
The Response of a Mechanical Oscillator Due to Swept and Dithered Excitation
}

\author{
R. Benjamin Davis ${ }^{1}$, R. Caitlyn Durham ${ }^{2}$, and Andrew M. Brown ${ }^{3}$ \\ NASA Marshall Space Flight Center, Huntsville, AL 35812
}

\begin{abstract}
A single degree-of-freedom oscillator subject to linearly swept and/or dithered excitation is considered. Dither refers to the variation of an excitation frequency about a given nominal-or primary - frequency. Dither in rocket engine turbopump shaft speeds can be an important consideration when analyzing the dynamic response of turbomachinery components such as turbine blades. Results indicate that the incorporation of dithered excitation into a fatigue analysis may extend the predicted fatigue life of the structure by a factor of two or more.
\end{abstract}

\section{Nomenclature}

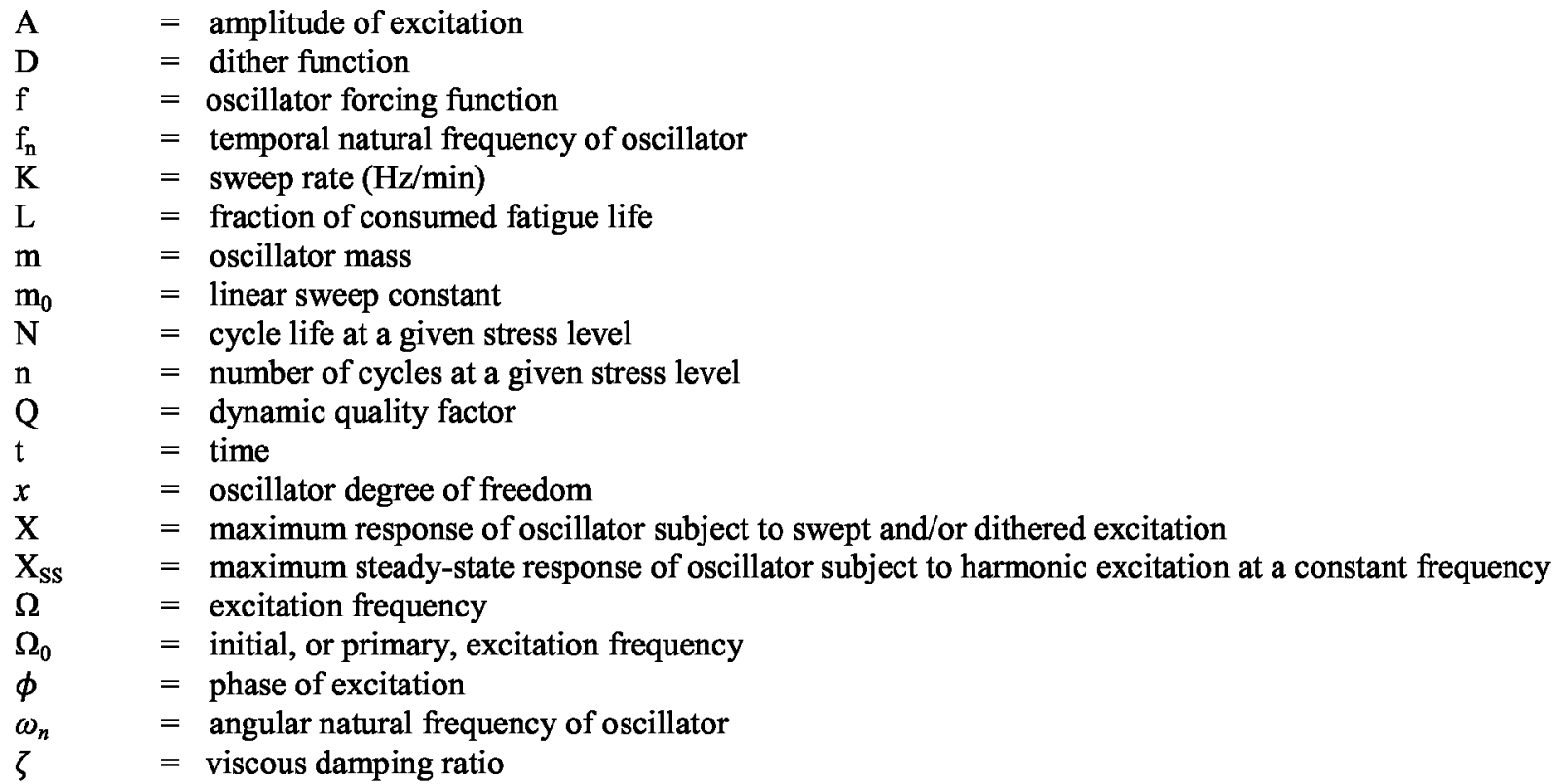

\section{Introduction}

$\mathrm{E}^{\mathrm{s}}$ ngineers responsible for the development of rocket engine turbomachinery have a long history of analyzing the structural dynamic response of individual turbopump components such as impellers or turbine blades. High cycle fatigue of these components is of concern especially if the component experiences prolonged excitation near a natural frequency. High-fidelity finite element models can do an excellent job of predicting component natural frequencies, but they will always have some associated error. Thus, when performing dynamic tests on turbomachinery components, it is common to linearly sweep the excitation frequency. This ensures that the component is excited - at some point during the sweep - near the natural frequencies of interest. It is important that

\footnotetext{
${ }^{1}$ Aerospace Engineer, Structural and Dynamics Analysis Branch, ER41, Member AIAA.

${ }^{2}$ Student Trainee, Structural and Dynamics Analysis Branch, ER41.

${ }^{3}$ Aerospace Engineer, Structural and Dynamics Analysis Branch, ER41, Senior Member AIAA.
} 
the test engineer specify a sweep rate that is sufficiently slow to achieve the maximum response from the component. A slow sweep rate thus enables the determination of accurate natural frequencies and damping values associated with the tested hardware.

While setting an appropriate sweep rate is important when testing components, dither about the primary excitation frequency is a consideration that arises when the turbopump is placed into service. The shaft speed of a turbopump is either a result of engine flow parameters or can sometimes be specified. In either case, the true instantaneous speed of the shaft will vary-or dither-about the commanded speed. For situations in which the nominal shaft speed results in a resonant response of a component, the presence of dither may reduce that response. Rocket engine analysts have historically cited dither as a mitigating factor when presenting dynamic stress and fatigue results, but the effects of dither have never been quantified for rocket engine applications.

Here, we consider the response of a single degree-of-freedom (SDOF) system subject to linearly swept or dithered excitation. The consideration of the swept case follows closely from the work of Lollock ${ }^{1}$ and is used primarily as a verification of the numerical methods employed herein. The consideration of dithered excitation is more novel and will be performed across a parameter space that is consistent with the operating experience of rocket engine turbopumps. The exploration of this parameter space gives an indication of the importance of dither when assessing the response of turbomachinery components.

\section{Methodology}

\section{A. Oscillator Model}

The linear SDOF oscillator is modeled with the familiar expression

$$
\ddot{x}+2 \zeta \omega_{n} \dot{x}+\omega_{n}^{2} x=\frac{f(t)}{m} .
$$

The excitation of this system is assumed to be sinusoidal and of the form

$$
f(t)=A \sin (\phi(t)),
$$

where $\phi(t)$ is the time varying argument, or phase, of the excitation. By definition, the instantaneous frequency of the forcing function is the time derivative of $\phi(t)$. Standard vibration analysis generally considers constant frequency excitation. In that case, $\phi(t)=\Omega t$ and it is clear that the frequency of excitation, $\Omega$, is equivalent to the time derivative of $\phi(t)$.

For a linearly swept excitation with dither, the time dependent frequency of the excitation can be expressed as

$$
\Omega(t)=\Omega_{0}+m_{0} t+D(t),
$$

where $\Omega_{0}$ is the initial - or primary-excitation frequency, $\mathrm{m}_{0}$ is the linear sweep constant, and $D(t)$ is the dither function. In most realistic applications, $D(t)$ will represent a random process with known statistical properties.

Recalling that instantaneous frequency is the time derivative of the phase, the phase function is found by integrating Eq. (3),

$$
\phi(t)=\int \Omega(t) d t=\int\left(\Omega_{0}+m t+D(t)\right) d t .
$$

The resulting function can then be substituted into Eq. (2) to generate the oscillator's forcing function.

\section{B. Numerical Approach}

The time-varying nature of the excitation frequency renders purely analytical solutions prohibitive. Instead, the state-space form of Eq. (1) is integrated numerically using a standard fourth/fifth-order (i.e., fourth-order accumulated error, fifth-order step error) Runge-Kutta algorithm that is based on the formulas presented by Cash and Karp ${ }^{2}$. This algorithm is especially well-suited to integrating differential equations that are driven with rapidly changing forcing functions. 
When subject to swept excitation, the oscillator response is reported in terms of a response fraction that is defined by

$$
\text { Response Fraction }=X / X_{s S}
$$

where $X$ is the maximum response of the oscillator subject to swept or dithered excitation and $X_{S S}$ is the maximum steady-state response of an oscillator subject to pure harmonic excitation at a constant frequency. For the lightly damped systems of concern, it can be shown that $X_{s s} \approx A /\left(2 \zeta m \omega_{n}^{2}\right)$, where $A$ is the amplitude of the harmonic excitation.

\section{Calculation of Life Factors}

The effects of rocket engine turbomachinery dither are quantified by comparing the fatigue life of a simulated turbopump component subject to harmonic excitation at a constant frequency to the fatigue life of the same component subject to dithered excitation. The ratio of these two fatigue life calculations is termed the life factor and represents the proportion of additional service life that is predicted when the effects of dither are considered in the analysis.

Here, fatigue life is calculated using Miner's Rule. Miner's Rule states that given a structure undergoing cyclical loading, each cycle will consume a certain amount of that structure's fatigue life ${ }^{3}$. By summing the amount of life consumed by each cycle, the total amount of life consumed can be determined. The fraction of consumed life can be found by

$$
L=\sum_{i=1}^{k} \frac{n}{N}
$$

where $n$ is the number of cycles at a given alternating stress level and $N$ is the number of cycles a given material is capable of withstanding at that alternating stress. The values of $\mathrm{N}$ are found using an $\mathrm{S}-\mathrm{N}$ curve for the material of interest. Eq. (8) is applied to calculate the fatigue life in both the case of the excitation at a constant frequency (i.e., the nominal fatigue life) and in the case of dithered excitation. The life factor is simply the ratio of these two values. That is,

$$
\text { Life Factor }=\frac{L_{\text {nominal }}}{L_{\text {dithered }}}
$$

\section{Results and Discussion}

\section{A. Linearly Swept Excitation}

To aid in the verification of the numerical methods, an independent reproduction of results reported by Lollock ${ }^{1}$ was performed. Figure 1 plots response fraction against a non-dimensional sweep rate given by

$$
\eta=\frac{Q^{2} K}{60 f_{n}^{2}}
$$

where $\mathrm{K}$ is the sweep rate $(\mathrm{Hz} / \mathrm{min})$ and $Q=1 / 2 \zeta$. The oscillator model that generated the curve in Figure 1 had a natural frequency of $1(\mathrm{~Hz})$ and viscous damping ratio of 0.01 . It can be shown, however, that when plotted against the non-dimensional parameter, $\eta$, the response fraction curves are insensitive to changes in oscillator natural frequency and damping, provided the damping ratio is low $(<0.03)$. 


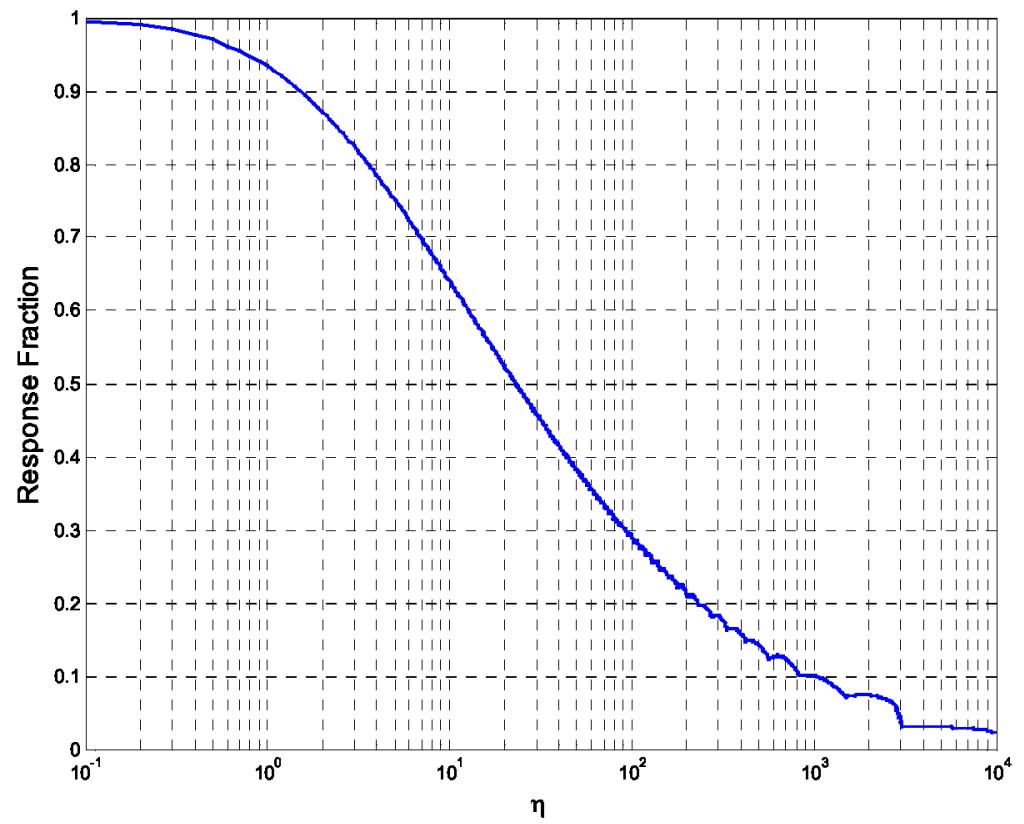

Figure 1: Response Fraction as a Function of Non-Dimensional Sweep Rate.

Figure 1 can be useful in the design of a dynamics experiment employing swept excitation. For an experimental set-up with a fixed sweep rate, Figure 1 allows one to determine how close the maximum response of the test article is to the maximum response that could be expected if the excitation were to dwell at a natural frequency of the article. Conversely, Figure 1 can be used to specify a sweep rate that will result in a desired response fraction. For example, one might wish specify a sweep rate that is slow enough to ensure that the test article will respond at $95 \%$ of its maximum response.

The response fraction curve shown in Figure 1 is entirely consistent with the results presented by Lollock ${ }^{1}$. This provides confidence in the numerical integration scheme being employed here.

\section{B. Dithered Excitation}

In this section, turbopump shaft speed data are used to quantify the effects of dither on the dynamic response of a SDOF system. In a typical flight profile, a turbopump is commanded to dwell at two or more different speeds. Figure 2 shows a representative turbopump shaft speed profile. Note the three distinct dwell periods as indicated by the red boxes. Turbomachinery components are typically subject to excitation at frequencies equal to certain integer multiples of a dwell frequency. If the component in question happens to have a natural frequency near a strong excitation frequency, then the component is susceptible to large amplitude response and possible failure due to high cycle fatigue.

The effects of turbomachinery dither are calculated for a number of turbopump shaft speed profiles each having multiple dwell periods. As evidenced by the dwell periods shown in Figure 2, the shaft frequency not only dithers, but may also drift up or down slightly over the course of a dwell period. To isolate the effects of dither, it is necessary to remove any drift from the data. This is accomplished by subtracting a five second moving average of the speed data from the raw speed data. The resulting signal represents the dither corresponding to a given dwell period. Figure 3 depicts the dither associated with the first dwell shown in Figure 2. Note that for this particular dwell, the excursions from the commanded speed are generally between $\pm 0.8(\mathrm{~Hz})$. 


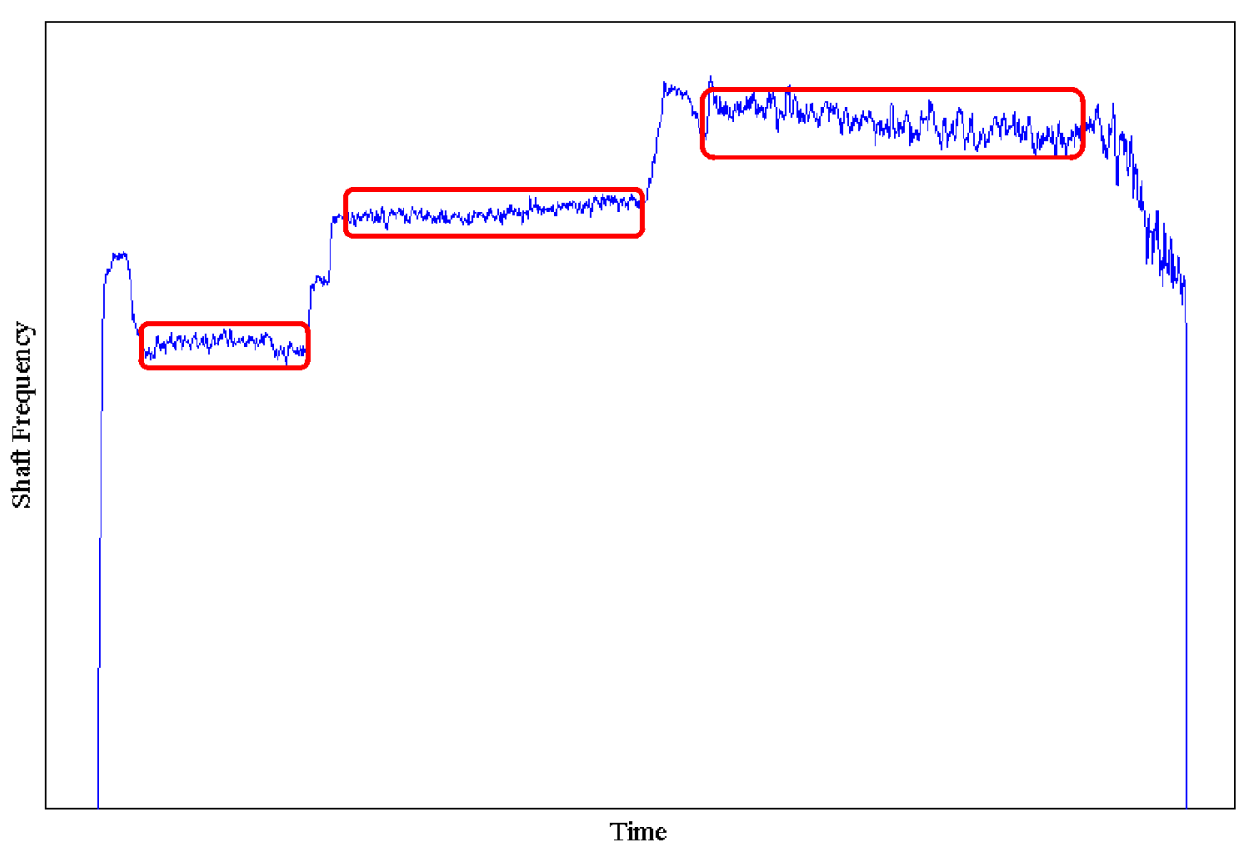

Figure 2: Instantaneous Shaft Frequency versus Time.

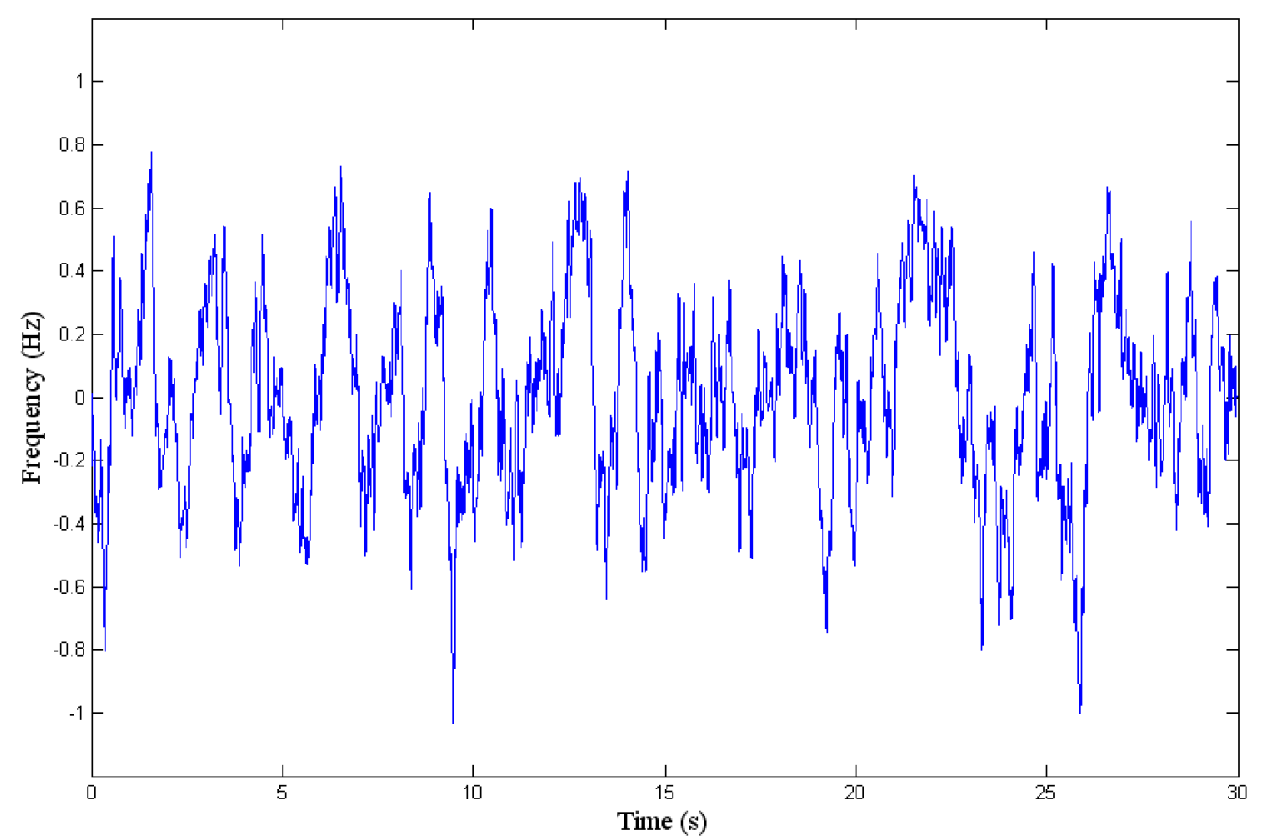

Figure 3: Shaft Frequency Dither versus Time.

Once the dither from a given dwell is isolated, the instantaneous frequency signal is generated. This is achieved by first adding the dither signal to an assumed primary frequency of the turbopump. The assumed primary frequency is chosen such that it is consistent with the typical commanded speeds of a given turbopump. Next, the instantaneous frequency signal is multiplied by the number of forcing cycles experienced by the component per a single revolution of the shaft. The resulting instantaneous frequency signal is then integrated according to Eq. (4) and applied as a forcing function to an SDOF oscillator as described in Section II A.

Figure 4 shows a one second sample of an instantaneous frequency signal. Here, the instantaneous frequency has been normalized by the assumed primary frequency of the turbopump. Figure 5 depicts the response fraction of the SDOF oscillator subject to excitation having the instantaneous frequency profile shown in Figure 4. Note that when the instantaneous frequency shown in Figure 4 strays from the primary frequency (indicated by the red line), a 
comparable decrease in oscillator response can be observed. Also observe the brief transient portion of the oscillator response. This transient behavior is discarded when calculating life factors. A viscous damping ratio of 0.0012 was used here. This value is consistent with damping ratios commonly found in (damper-less) turbine flow-path hardware.

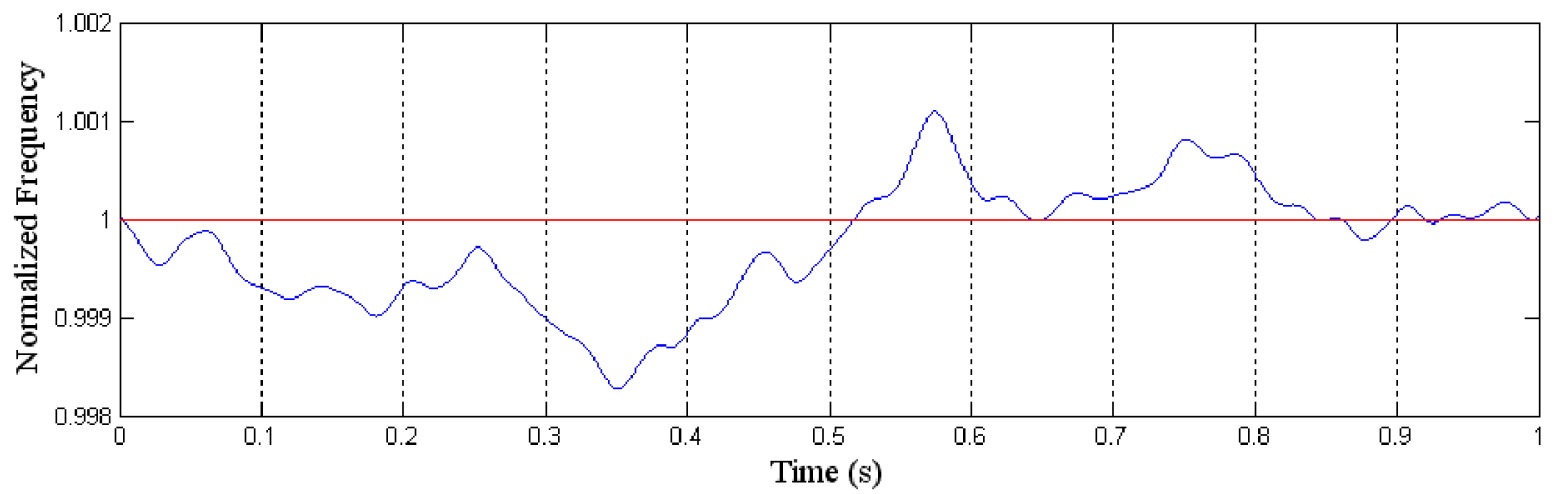

Figure 4: Instantaneous Frequency versus Time

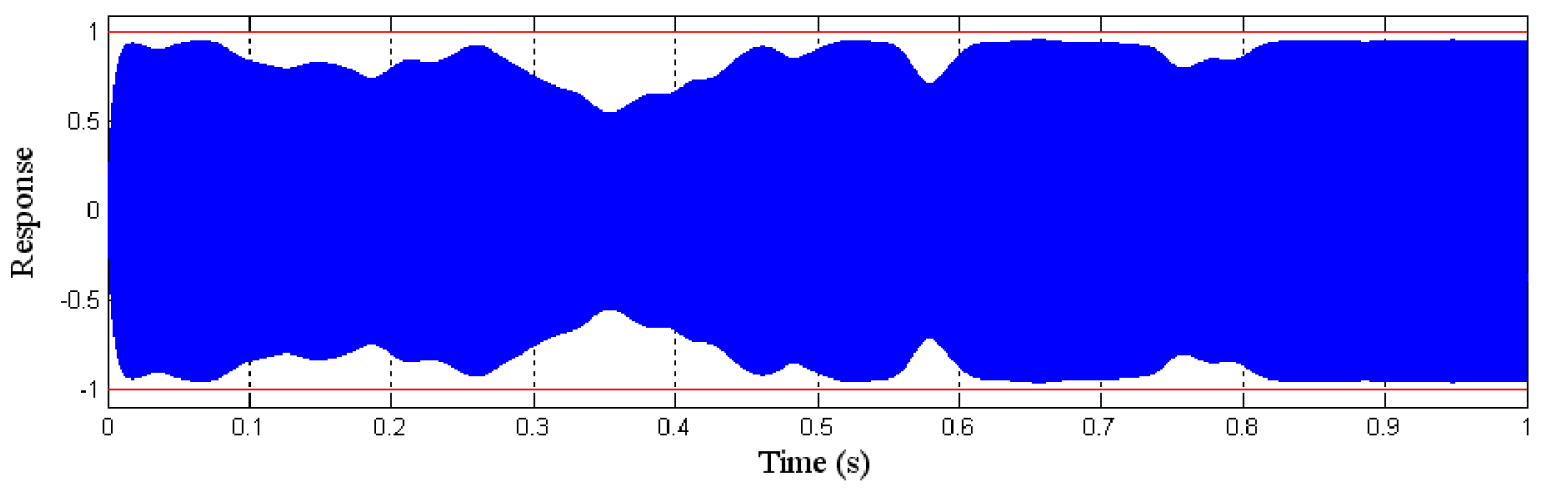

Figure 5: Oscillator Response versus Time

Figure 6 shows a scatter plot of the oscillator amplitudes shown in Figure 5. The amplitudes are plotted against their corresponding instantaneous frequencies and are overlaid on the steady-state response curve of the corresponding SDOF oscillator. Notice that the response amplitudes fall closely along the top portion of the response curve. This indicates that the transient nature of the excitation does not cause the oscillator to deviate greatly from the theoretical steady-state response. In such cases it might therefore be possible to simplify the analysis approach used here. Instead of numerically integrating the oscillator equation of motion subject to a dithered excitation signal, one could approximate oscillator response amplitude by substituting instantaneous forcing frequency information into the forced response function of the SDOF system. However, depending on the characteristics of the oscillator and the dithered excitation, this simplification may not always be appropriate. 


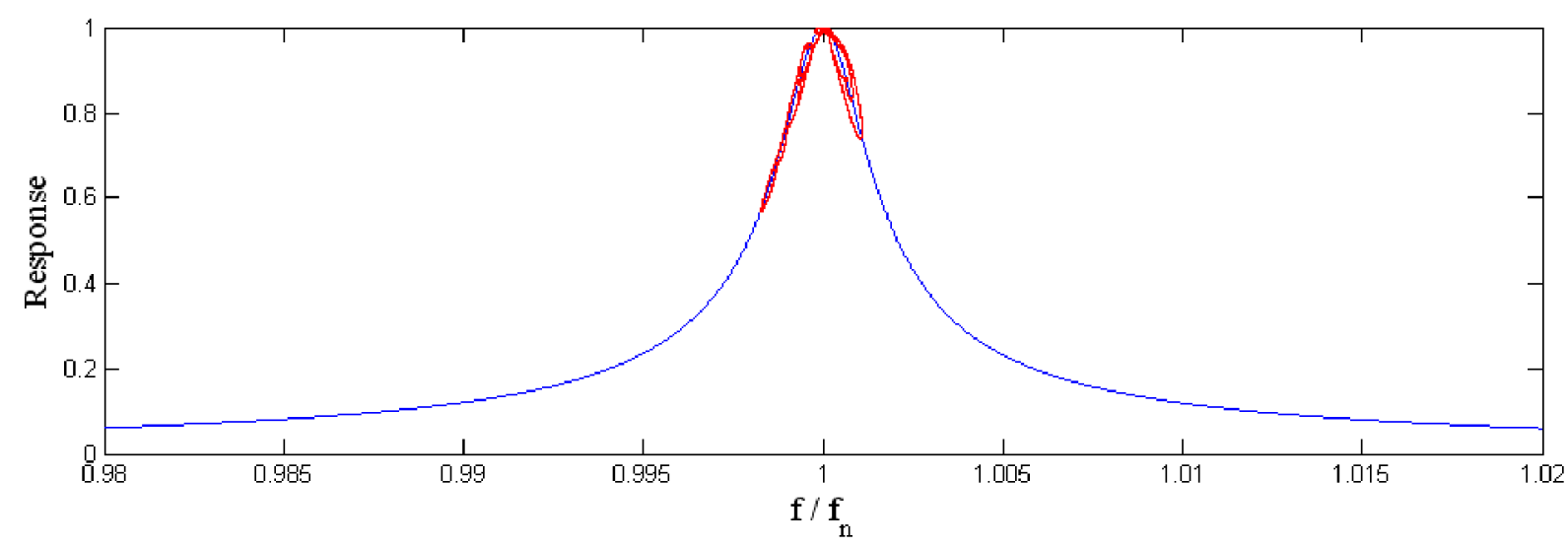

Figure 6: Dithered Response as a Function of Normalized Frequency.

The above-mentioned simplification is not appropriate in the case illustrated by Figure 7. Here, the response amplitudes do substantially deviate from the theoretical curve. Figure 7 depicts both analytical (left) and experimental (right) response scatter plots. The experimental responses represent strain gage data obtained during the Space Shuttle Orbiter flowliner cracking investigation. The flowliner is subject to strong excitation at four times the pump running speed and is excited near a resonant frequency of approximately $1,070(\mathrm{~Hz})$. The analytical plot was created for a SDOF system with this same resonant frequency and was also excited at four times the pump running speed. It should be noted, however, that the dither data used to excite the analytical system was obtained from a turbopump that is different from the one that was used in conjunction with the flowliner system. Nevertheless, the two plots demonstrate good agreement, with both sets of response amplitudes filling out a large portion of the domain enclosed by the frequency response curve. Contrast this with Figure 6 where the oscillator is subject to excitation at 74 times the pump running speed. As will be discussed in the next section, oscillators subject to a low number of forcing cycles per revolution are less able to track the changes in excitation frequency. As a result, the response amplitudes of these oscillators can stray significantly from the steady-state response curve. Figure 7 also illustrates an important point concerning damping. The response curve in the experimental scatter plot was created by adjusting damping ratio such that the vast majority of the response data falls just inside the response curve. The damping ratio used to create this curve was 0.0014. The same response curve is plotted for the analytical case. Note that a significant portion of the analytical response data falls outside the response curve. This indicates that attempting to estimate damping by fitting a response curve around a set of experimental responses may result in an over-prediction of damping ratio. Thus, the actual damping ratio associated with the flowliner data shown here is likely even less than 0.0014 .
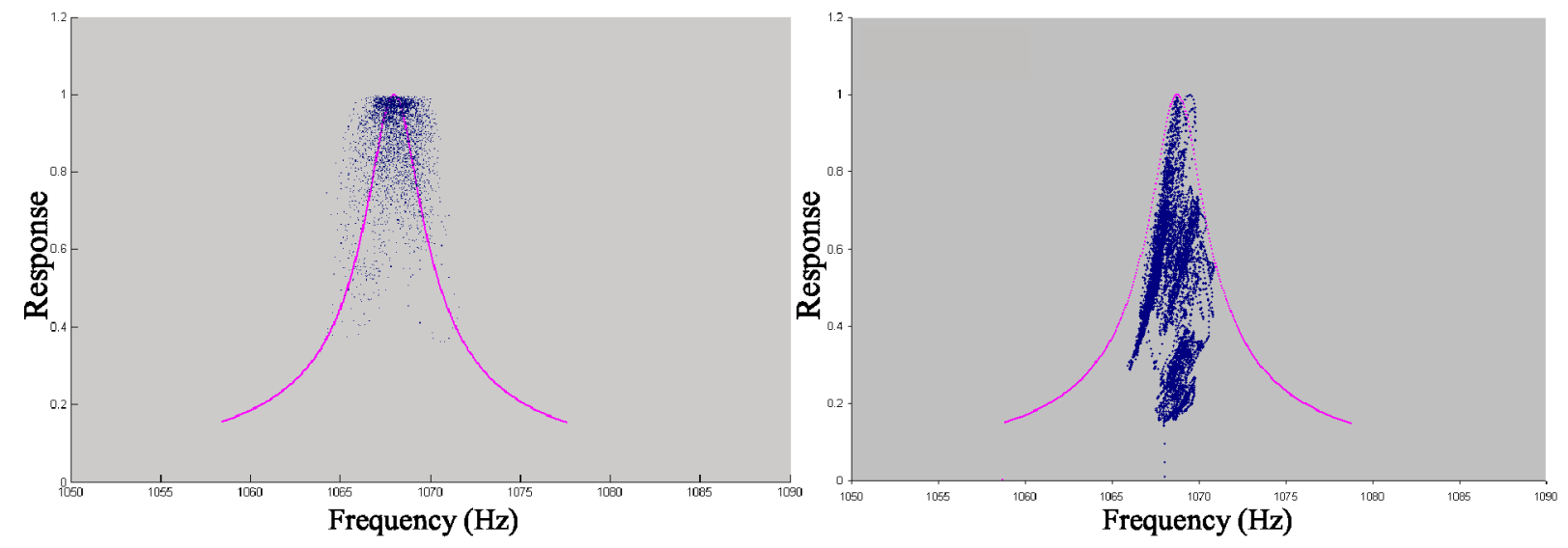

Figure 7: Analytical and Experimental Response Scatter Plots for Similar Turbopumps.

Life factors have been calculated using shaft speed data collected during tests of two different rocket engine turbopumps. A total of 18 life factors have been calculated with each life factor corresponding to a single dwell 
period. The 18 cases represent at total of 1,412 seconds of data and the same baseline damping ratio was used for all cases. However, due to different design characteristics, the primary shaft frequency and the number of forcing cycles per revolution were different for the two turbopumps. In all cases, the forcing function was sampled at 50 times the natural period of the oscillator and a viscous damping ratio of 0.0012 was used. The life factors calculated for the first turbopump ranged from 2.02 to 5.64, with a weighted average of 3.57 , while those calculated for the second turbopump ranged from 2.62 to 8.56 , with a weighted average of 4.69. This indicates that for lightly damped structures used in typical rocket engine turbopumps, it is reasonable to expect the predicted fatigue life of the structure to be two to three times longer when the effects of dither are considered.

\section{Sensitivity Studies}

When faced with the analysis of a new turbopump, a complete set of sensitivity studies enables the analyst to decide if it is worthwhile to incorporate effects of dither in the analysis. To this end, several sensitivity studies have been devised to observe how calculated life factors change with incremental changes to individual parameters.

All sensitivity studies were performed by calculating life factors over a single ten second representative sample of turbopump speed data. In each study, the single parameter was varied while all other parameters were held constant at their baseline levels. In the first test, the effects of damping ratio were studied by varying $\zeta$ values from 0.001 to 0.01 . Figure 8 plots life factor versus damping ratio. The curve demonstrates that the effects of dither are important for very lightly damped structures. Judging from Figure 8, it appears that accounting for the effects of dither may only be worthwhile if the structure of interest has damping ratios less than approximately 0.003 .

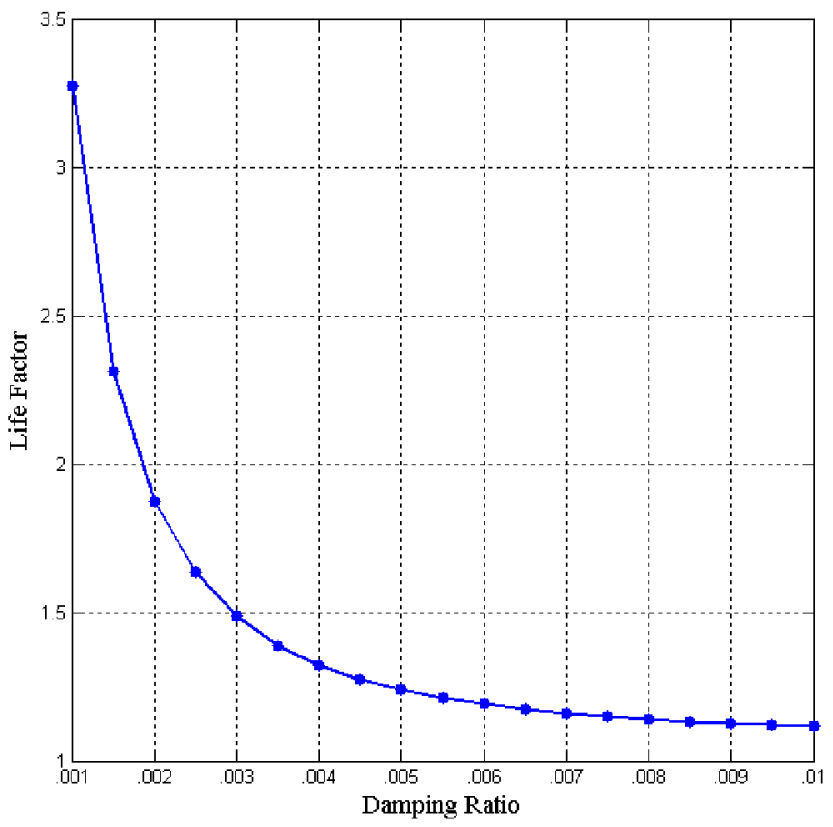

Figure 8: Life Factor versus Damping Ratio.

The fact that life factor decreases with increasing damping ratio can be understood by considering how the forced response curve of a SDOF system changes with damping ratio. Increases in damping result in forced response curves with wider peaks. Thus, when damping is relatively high, a fixed frequency excursion from the primary excitation frequency will result in a smaller relative reduction in amplitude. Figure 9 illustrates this point. Consider the forced response curves of a SDOF system where $\zeta=0.001$ and one where $\zeta=0.01$. Each forced response curve is normalized by its respective peak value. Consider forcing at a normalized frequency of unity and at a normalized frequency of 1.005. At a normalized frequency of unity, both normalized response amplitudes will be very near their respective maxima. At a normalized frequency of 1.005 , the response amplitude will be just $20 \%$ of its peak value in the case where $\zeta=0.001$. However, in the case where $\zeta=0.01$, a normalized frequency of 1.005 results in a response amplitude that is almost $90 \%$ of the peak value. The fact that forced response curves have lower peak values for higher levels of damping is immaterial to this discussion since the two fatigue life values used 
in the calculation of life factor (see Eq. (9)) require the use of the same damping ratio. Thus, the calculation of life factor is independent of the actual amplitude response of the component. Rather, it is a measure of the response of a system to dithered excitation relative to the response of the same system subject to constant frequency excitation.

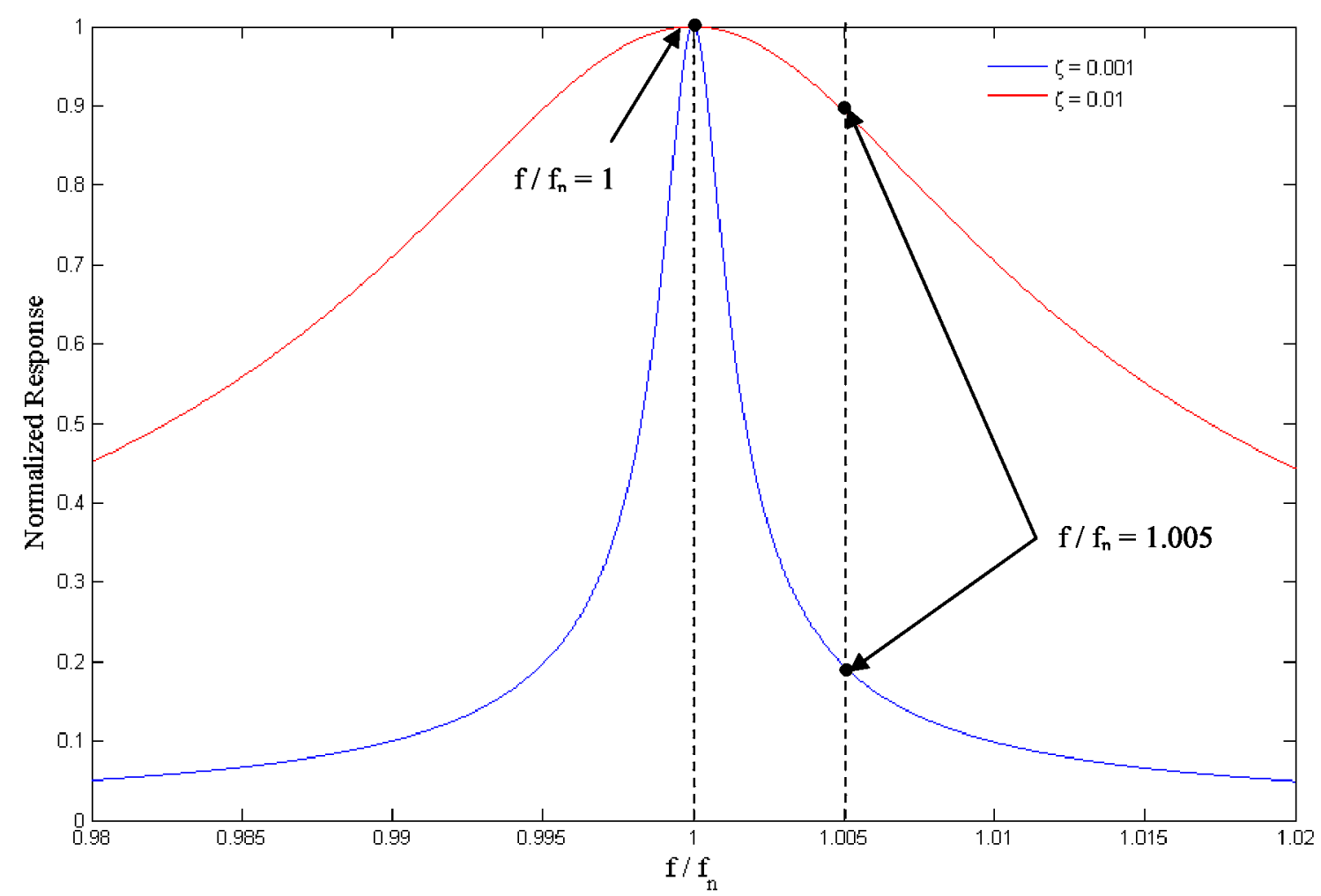

Figure 9: Normalized Frequency Response for Two Values of Damping Ratio.

As discussed in previous sections, a turbopump component is subject to strong excitation at frequencies corresponding to critical integer multiples of the shaft speed. This value will now be referred to as forcing cycles per revolution (FPR). The sensitivity of life factor to changes in FPR was tested for FPR values ranging from one to 100. The results of the study are shown in Figure 10. This study indicates that for FPR values greater than 40, life factor is largely insensitive to changes in FPR value. This finding can be explained by considering the amplitude response of oscillators subject to excitation with different FPR values. Figure 11 depicts these responses. Note that for low FPR, the oscillator is less able to track rapid changes in excitation frequency and the resulting response amplitude curve is relatively flat. As FPR increases, the oscillator executes many more full oscillations per shaft revolution. Thus, its period is fast relative to the rate of change of excitation frequency and it is much more able to track the changes in excitation frequency. Note also that there is very little difference between the cases where FPR $=50$ and FPR $=100$. This helps explain why the life factor calculations are largely insensitive above FPR values of 40 . 


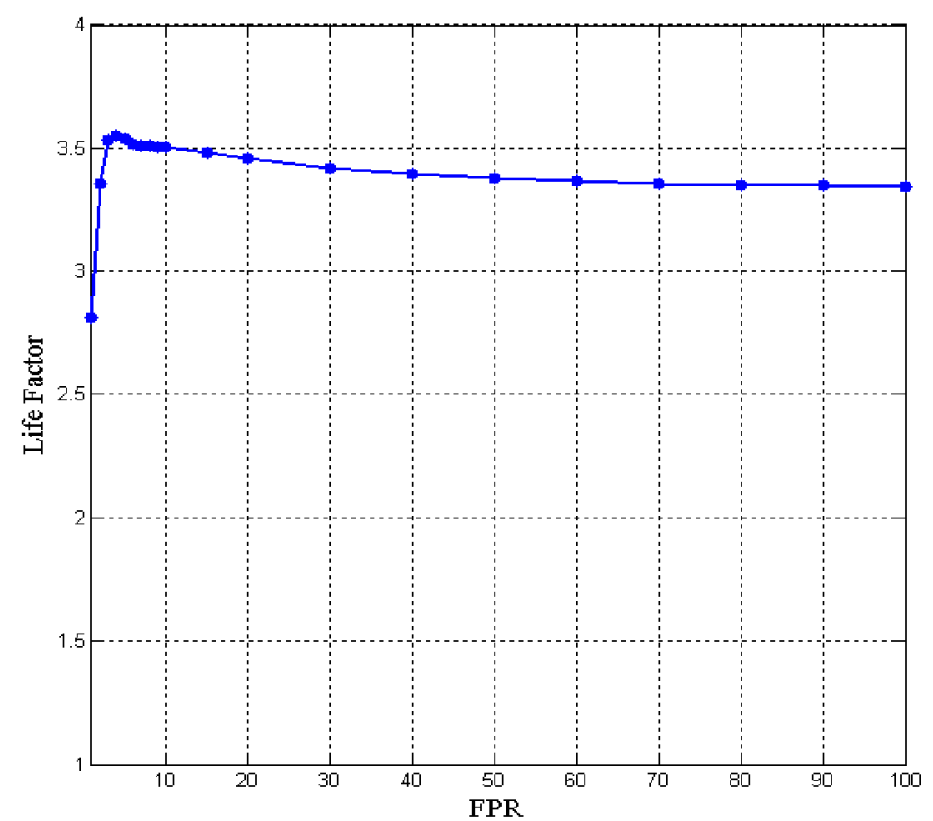

Figure 10: Life Factor as a Function of FPR.

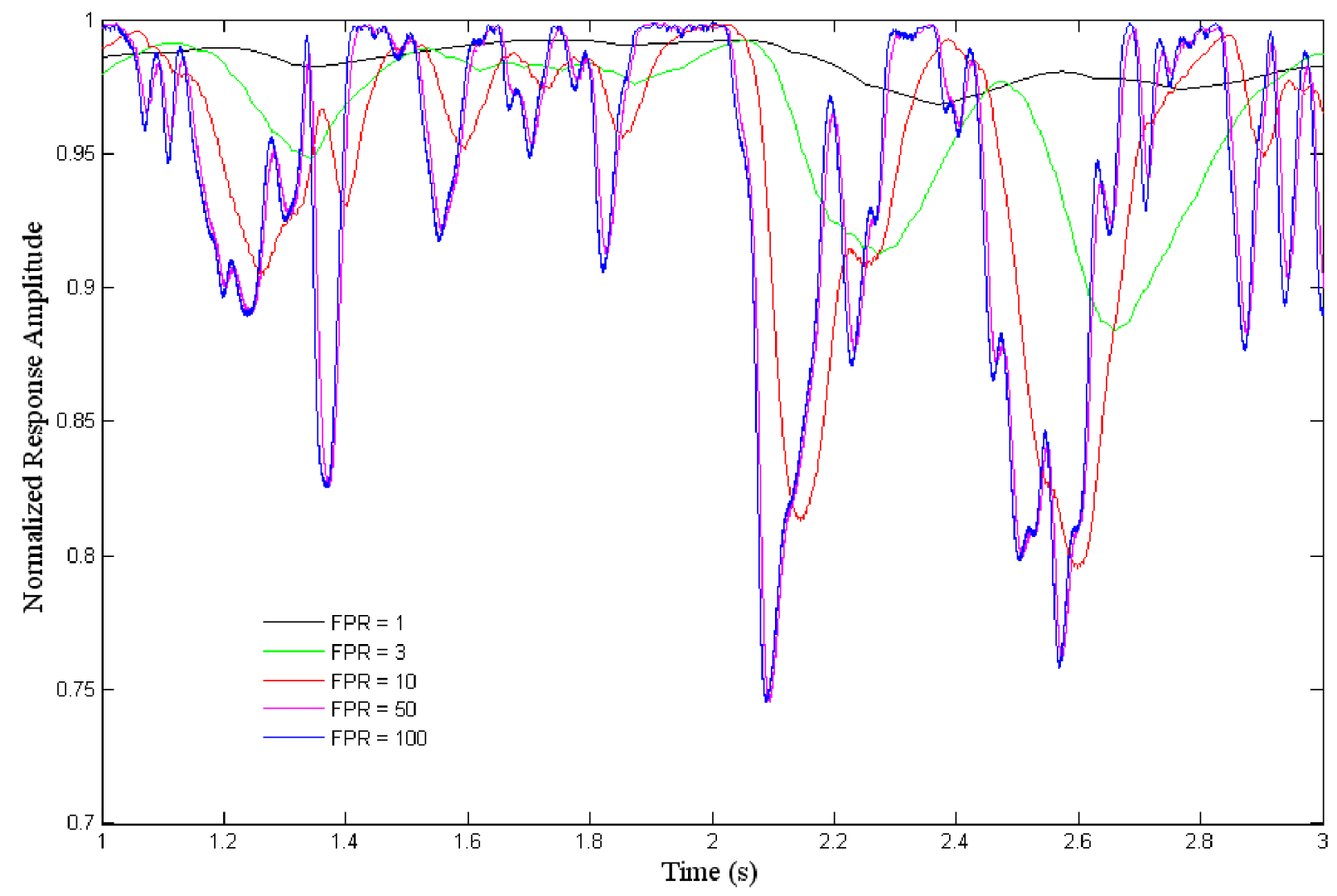

Figure 11: Response Amplitude versus Time for Various Values of FPR.

The effects of varying the primary shaft frequency of the turbopump were also studied. The primary shaft frequency was investigated over the range of $130(\mathrm{~Hz})$ to $550(\mathrm{~Hz})$ and Figure 12 plots life factor over this range. The trend observed in Figure 12 can be explained by considering the fact that the dither (i.e., the extent of frequency excursions from the primary frequency) is fixed regardless of primary frequency, so the forcing frequency 
excursions are relatively more severe when the primary shaft speed is lower. Relatively larger forcing frequency excursions result in lower response amplitudes which, in turn, result in larger life factors.

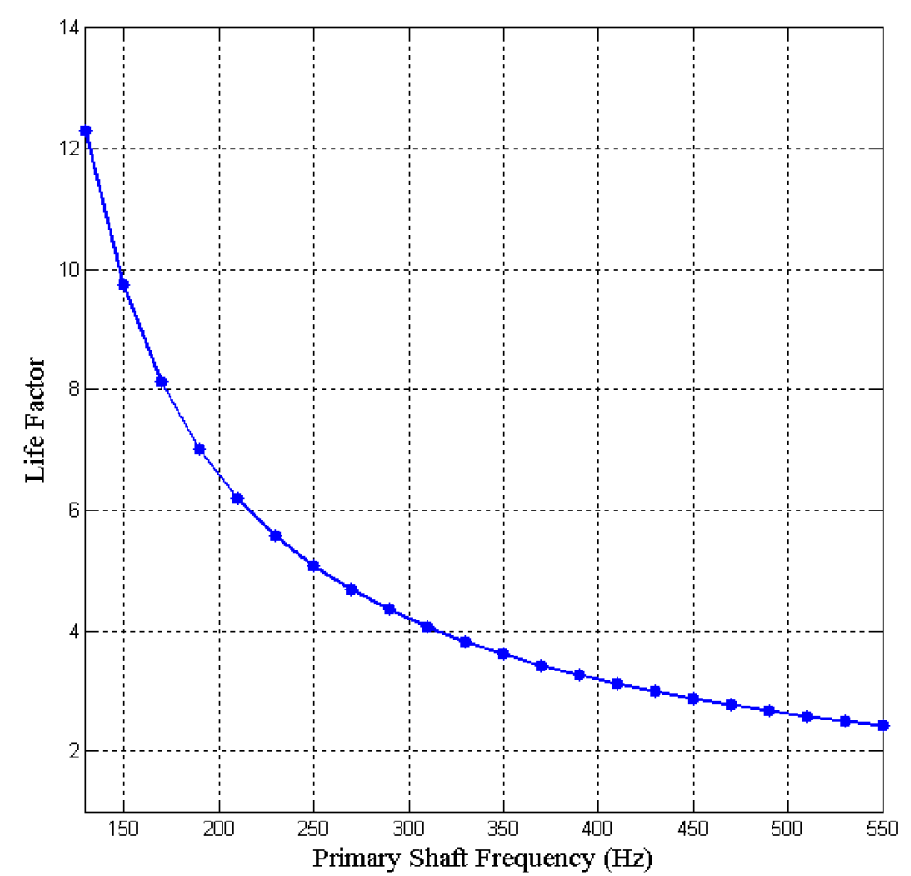

Figure 12: Life Factor as a Function of Primary Shaft Frequency.

The final sensitivity study was aimed at determining the appropriate sampling rate for the excitation signal. To accurately determine oscillator response peaks, it is necessary to sample the forcing function many times per forcing cycle. In this study, the number of samples per forcing cycle were varied from 10 to 70 and Figure 13 shows the life factor as a function of samples per forcing cycle (i.e., sampling multiplier). Note the good convergence of life factor values for sampling multipliers greater than 40 . For the baseline cases reported here, a sampling multiplier of 50 was used. The study indicates that the life factors calculated using a sampling multiplier of 50 are approximately $2.5 \%$ higher than those calculated using a sampling multiplier of 70 . Thus, the life factors reported at the end of the previous section may represent slight over-predictions. 


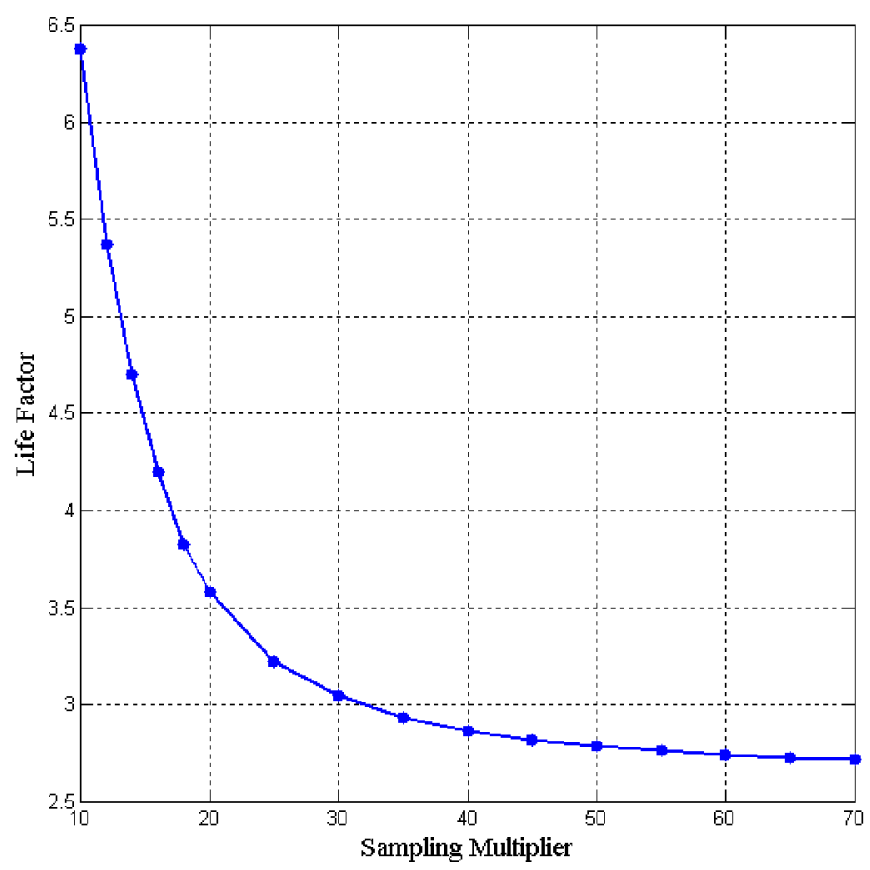

Figure 13: Life Factor versus Sampling Multiplier.

\section{Conclusion}

It has long been understood that conventional finite life fatigue calculations on rocket engine turbomachinery components carry some additional conservatism in that they do not consider the effects of turbopump speed dither. However, this additional conservatism has never been quantified. Using actual turbopump shaft speed data and a simulated structural component with representative properties, it was determined that finite life predictions can be at least two times longer if the effects of dithered excitation are considered. In certain cases, a two times longer fatigue life prediction may represent the difference between judging a component to be structurally adequate or not. Since every turbopump design is different, it is unreasonable to assume that the consideration of dither will always result in life predictions that are at least two times longer. Nevertheless, the life factor results and the sensitivity studies reported here should provide the analyst the information necessary to determine if the effects of turbopump dither are an important analysis consideration.

\section{References}

1 Lollock, J. A., "The Effect of Swept Sinusoidal Excitation on the Response of a Single Degree-of-Freedom Oscillator," $43^{\text {rd }}$ AIAA/ASME/ASCE/AHS/ASC Structures, Structural Dynamics, and Materials Conference, AIAA 2002-1230, Denver, CO, 2002.

${ }^{2}$ Cash, J. R., and Karp, A. H., "A Variable Order Runge-Kutta Method for Initial Value Problems with Rapidly Varying Right-Hand Sides," ACM Transactions on Mathematical Software, Vol. 16, No. 3, 1990, pp. 201-222.

${ }^{3}$ Juvinall, R. C., and Marshek, K. M., Fundamentals of Machine Component Design, $3^{\text {rd }}$ ed., Wiley, New York, 2000 , Chap. 8. 


\section{The Response of a Mechanical Oscillator Due to Swept and Dithered Excitation}

\section{Ben Davis, Caitlyn Durham, and Andy Brown NASA Marshall Space Flight Center}

51st AIAA/ASME/ASCE/AHS/ASC Structures, Structural Dynamics and Materials Conference April 12-15, 2010 


\section{Introduction}

- A single degree-of-freedom (SDOF) oscillator subject to linearly swept and/or dithered excitation is considered.

- The instantaneous rotational speed of the turbopump shaft varies - or dithers - around the set rotational speed.

- When the nominal shaft speed causes the part to reach resonance, dither may:

1. Reduce the maximum response of the part

2. Extend the part's predicted fatigue life 


\section{Methodology}

The linear SDOF oscillator is modeled with the expression

$$
\ddot{x}+2 \zeta \omega_{n} \dot{x}+\omega_{n}^{2} x=\frac{f(t)}{m}
$$

where the forcing function $f(t)$ is sinusoidal, and

$$
f(t)=A \sin (\phi(t))
$$

$\varphi(t)$ is the time varying argument of the excitation. The instantaneous frequency of the forcing function is the time derivative of $\varphi(t)$. Standard vibration analysis considers constant frequency excitation. In that case, $\varphi(t)=\Omega t$ and the frequency of excitation, $\Omega$, is equivalent to the time derivative of $\varphi(t)$.

$$
\phi(\mathrm{t})=\Omega_{0}+m t+D(t)
$$

where $\Omega_{0}$ is the primary excitation frequency, $m$ is the linear sweep constant, and $\mathrm{D}(\mathrm{t})$ is the dither function. 


\section{Methodology}

- The phase function is found by integrating $\varphi(t)$

$$
\phi(t)=\int \Omega(t) d t=\int\left(\Omega_{0}+m t+D(t)\right) d t
$$

- Substituting into $f(t)=A \sin (\phi(t))$ will generate the oscillator's forcing function. 


\section{Linearly Swept Excitation NAss}

- An reproduction of results reported by Lollock ${ }^{1}$ was performed. Natural frequency $=1(\mathrm{~Hz})$ and viscous damping ratio $=0.01$.

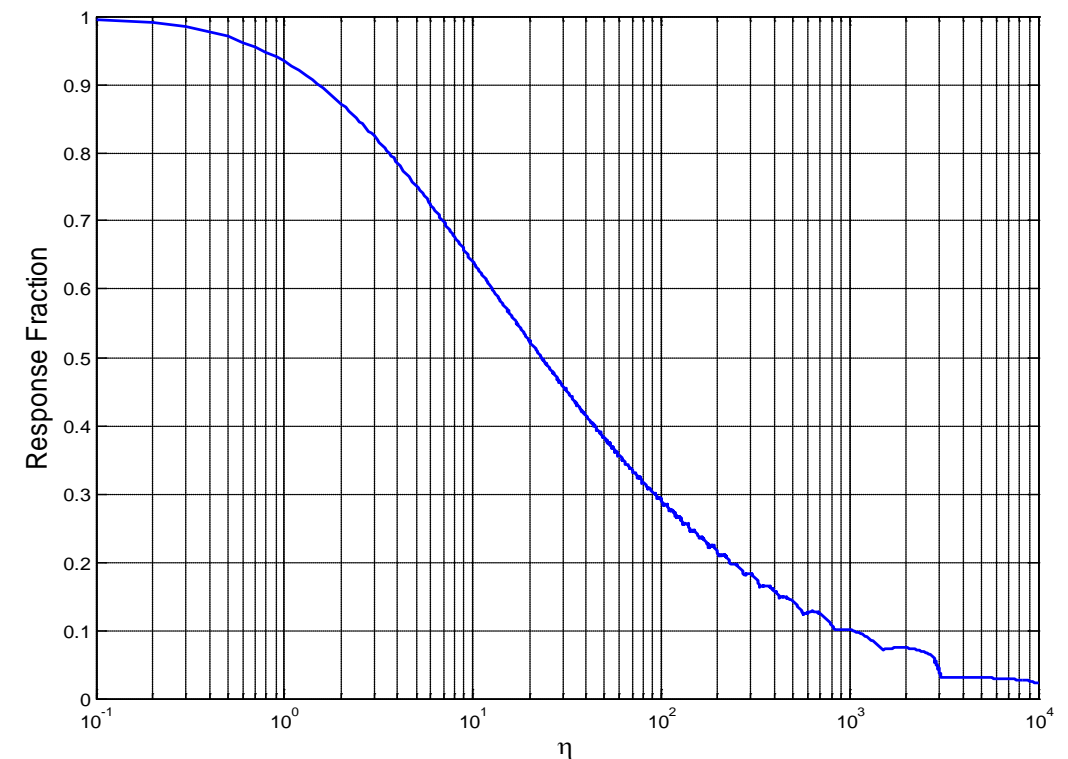

- When plotted against $\eta$, the response fraction is insensitive to oscillator natural frequency and damping. (damping ratio $<0.03$ )

- Response fraction is entirely consistent with Lollock ${ }^{1}$. 


\section{Test Data}

- The effects of dither are calculated for turbopump shaft speed profiles, each having multiple dwell periods.
- Moving average of turbopump shaft speed is removed from total speed signal to produce dither signal
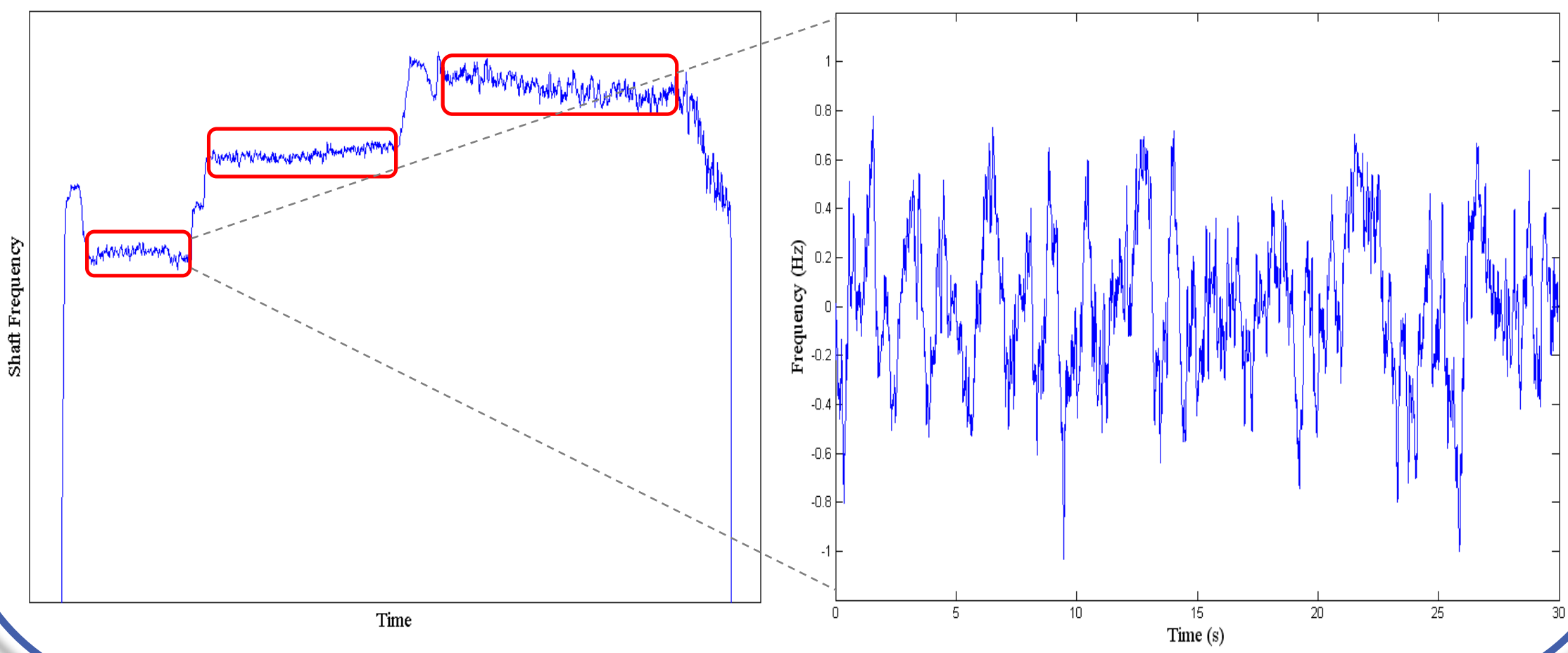


\section{Sample Oscillator Response}

- Dither signal is divided into one second segments that will be analyzed individually
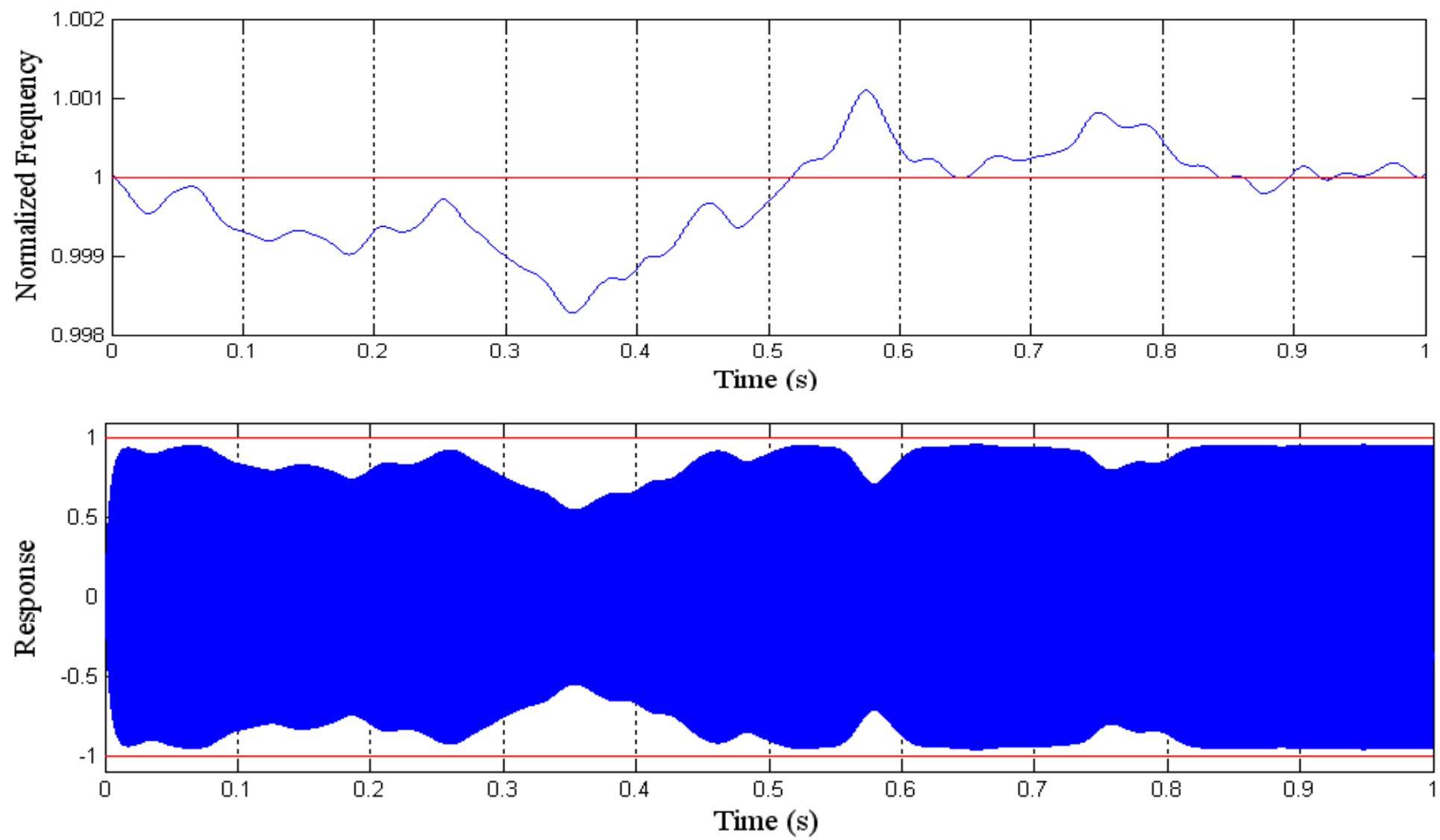

- When the instantaneous frequency strays from the primary frequency, a comparable decrease in oscillator response can be observed. 


\section{Sample Oscillator Response}

- The oscillator response amplitudes are plotted with the corresponding instantaneous frequencies and overlaid on the response curve of a SDOF oscillator.

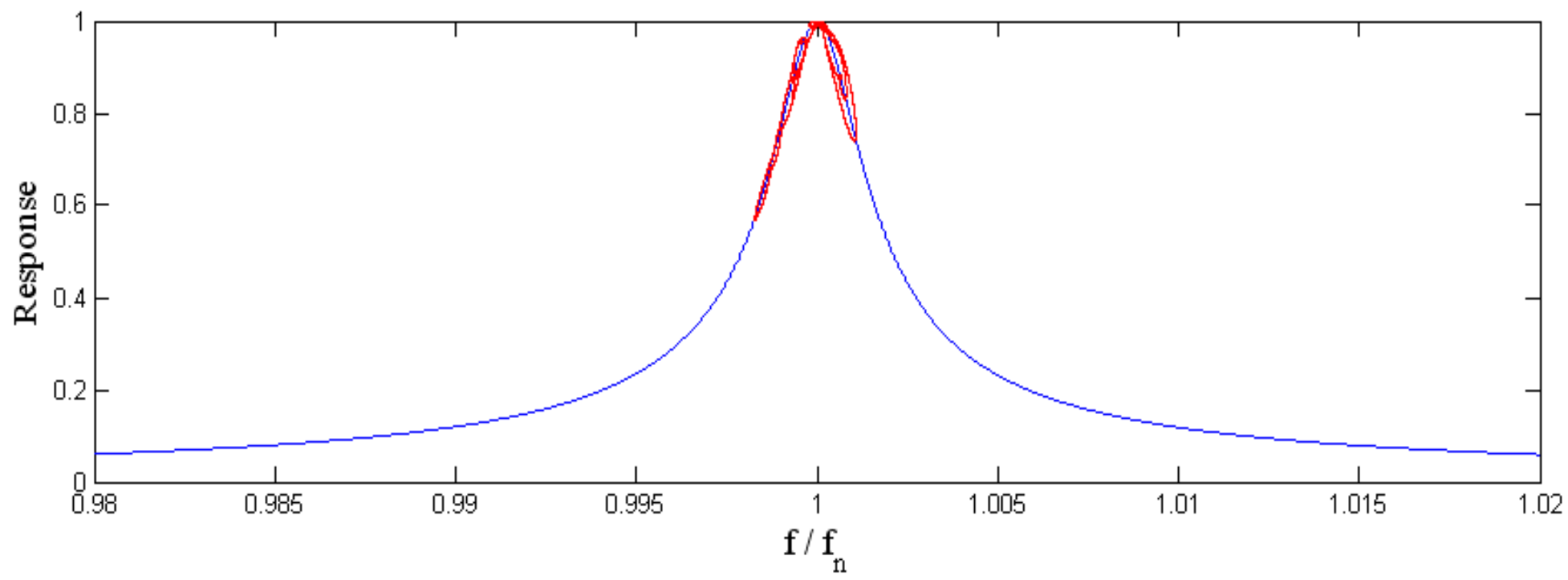

- The response amplitudes fall closely along the top portion of the response curve. 


\section{Response Comparison}

- Response amplitudes deviate from the theoretical curve.

- Experimental response curve created by adjusting damping ratio so that the response data is inside the curve

- May result in an over-prediction of damping ratio.

\section{Analytical Response}

- From Sample Oscillator response data

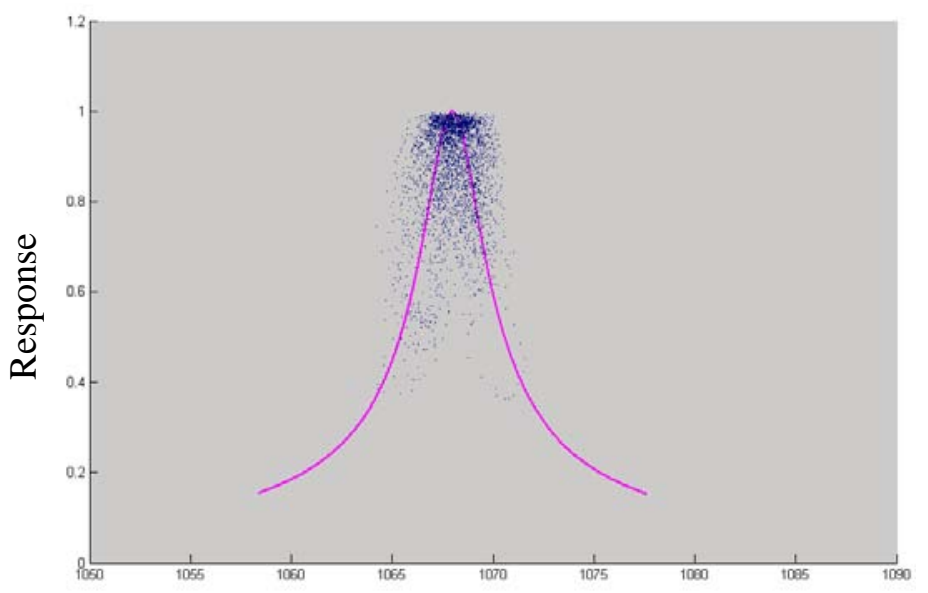

Frequency (Hz)

\section{Experimental Response}

- From Space Shuttle Orbiter flowliner cracking investigation.

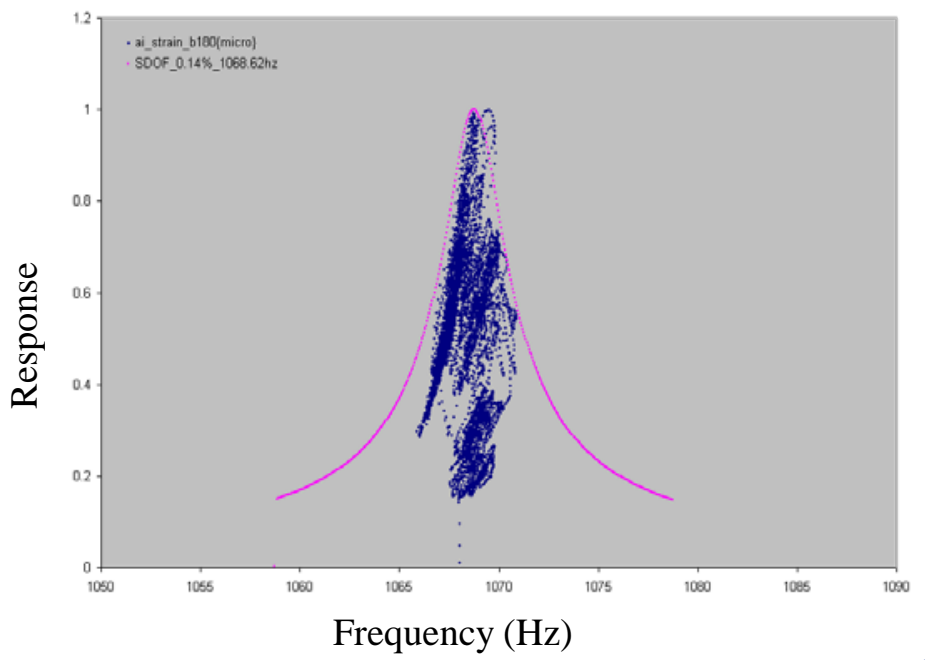




\section{Life Factor Calculation}

- The life factor quantifies the effects of dither.

- Life factor represents the proportion of additional service life, when dither is considered.

- Ratio of nominal fatigue life to dithered fatigue life.

- Miner's Rule: given a structure undergoing cyclical loading, each cycle will consume a certain amount of that structure's fatigue life ${ }^{2}$ 


\section{Life Factor Calculation}

- The fraction of consumed life can be found by summing the amount of life consumed by each cycle

$$
\boldsymbol{L}=\sum_{\mathrm{i}=1}^{\mathrm{k}} \frac{\mathbf{n}}{\mathrm{N}}
$$

where $n=$ number of cycles at a given stress level and $N=$ cycle life at a given stress level.

- The summation is applied to calculate the fatigue life in the case of the excitation at a constant frequency and in the case of dithered excitation.

- The life factor is the ratio of nominal life to dithered life.

$$
\text { LifeFactor }=\frac{L_{\text {Nominal }}}{L_{\text {dithered }}}
$$




\section{Results}

- 20 life factors calculated from two different turbopumps

- Each life factor corresponds to a single dwell period.

- The 20 calculated life factors represent 1622 seconds of data.

- Range of calculated life factors

- Turbopump 1: 2.03 - 5.64 (Weighted Ave. = 3.82)

- Turbopump 2: 2.62 - 8.56 (Weighted Ave. = 5.17)

- Predicted life may be two or more times longer when dither is considered. 


\section{Sensitivity Studies}

- Sensitivity studies observe how life factors change with incremental changes to individual parameters.

- Damping ratio

- Forcing cycles per revolution (FPR)

- Primary shaft frequency

- Number of samples per forcing cycle (sampling multiplier)

- 10 second representative sample 


\section{Damping Ratio}

- Damping ratio values range from 0.001 to 0.01

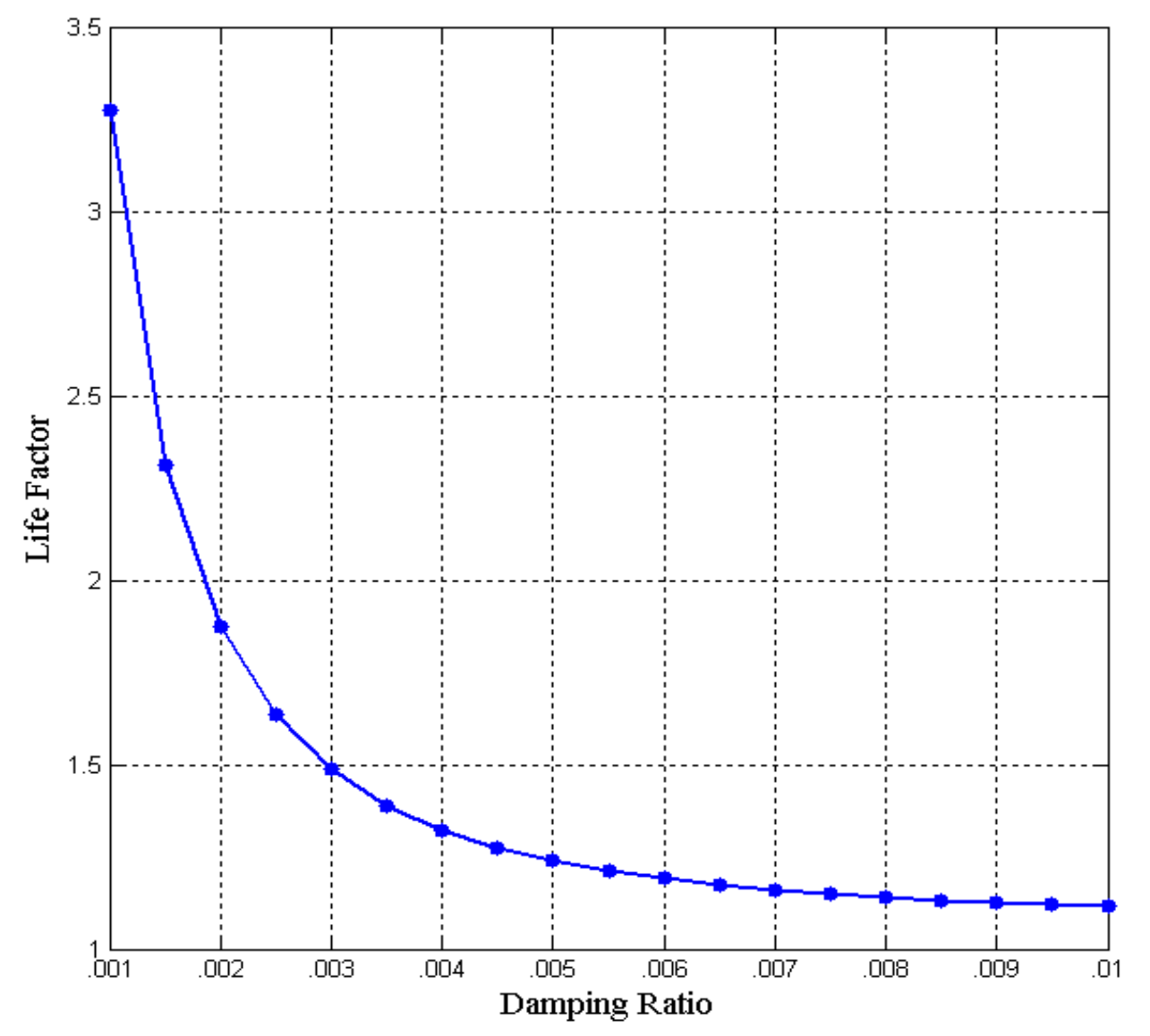




\section{Damping Ratio}

- Increases in damping result in forced response curves with wider peaks. When damping is high, a fixed frequency excursion from the primary excitation frequency results in a smaller relative reduction in amplitude.

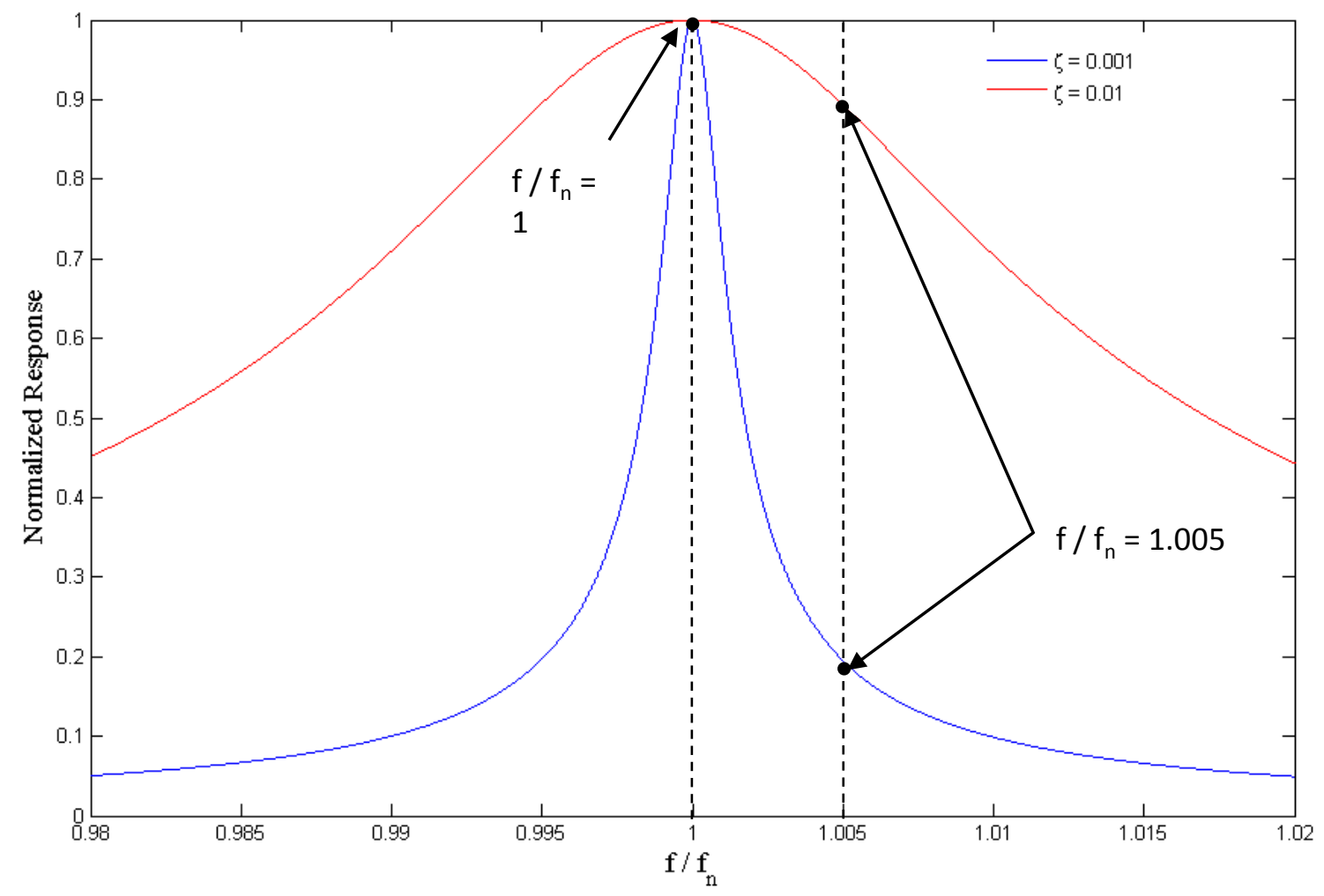




\section{FPR}

- A turbopump component is subject to strong excitation at frequencies corresponding to critical integer multiples of the shaft speed.

- FPR values tested from one to 100.

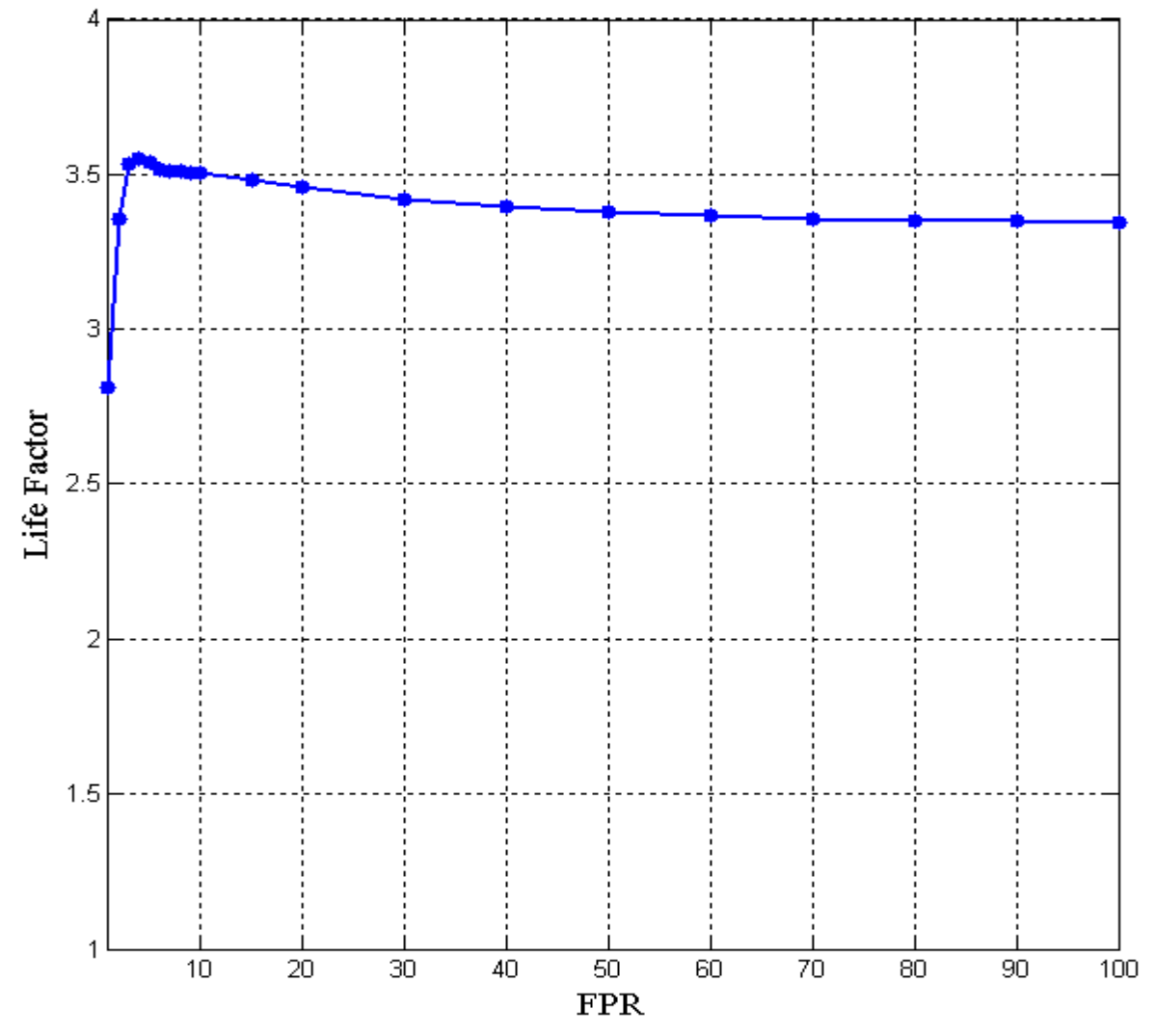




\section{FPR}

- For FPR values greater than 40, life factor is largely insensitive to changes in FPR value.

- Consider the amplitude response of oscillators subject to excitation with different FPR values.

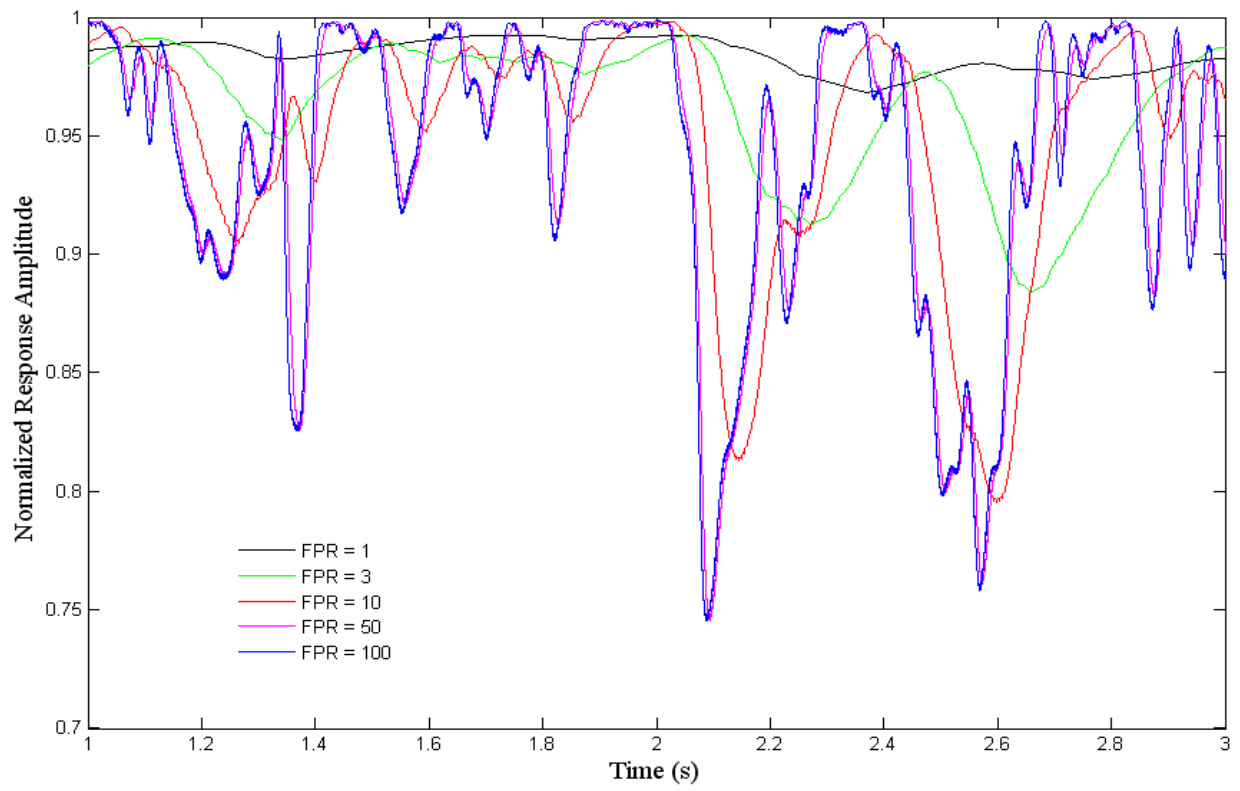

- For low FPR, the oscillator is less able to track rapid changes in excitation frequency and the resulting response amplitude curve is relatively flat.

- More full oscillations at higher FPR. 


\section{Primary Shaft Frequency}

- Primary shaft frequency range is $130(\mathrm{~Hz})$ to $550(\mathrm{~Hz})$

- Dither is fixed regardless of primary frequency,

- Forcing frequency excursions are relatively more severe when the primary shaft speed is lower.

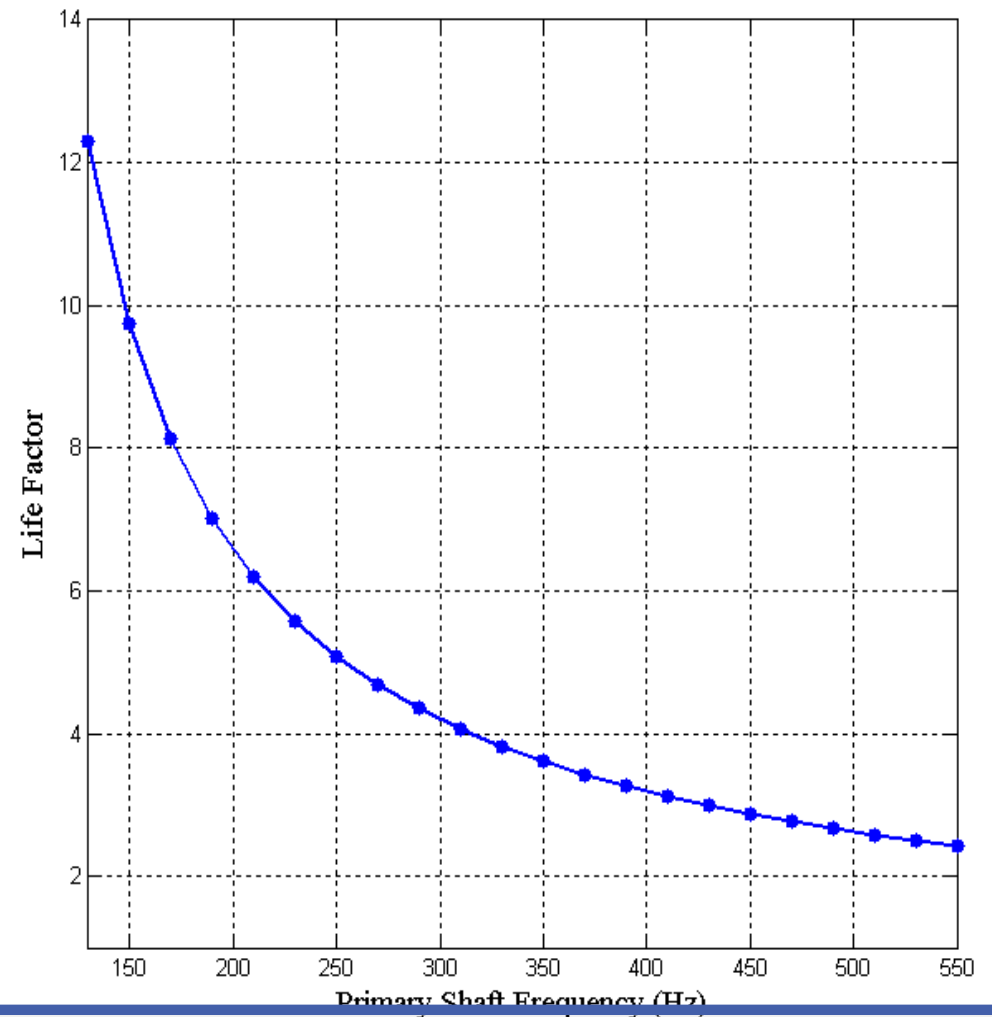




\section{Sampling Multiplier}

- Range: 10 to 70

- For baseline case, number of samples per forcing cycle was 50.

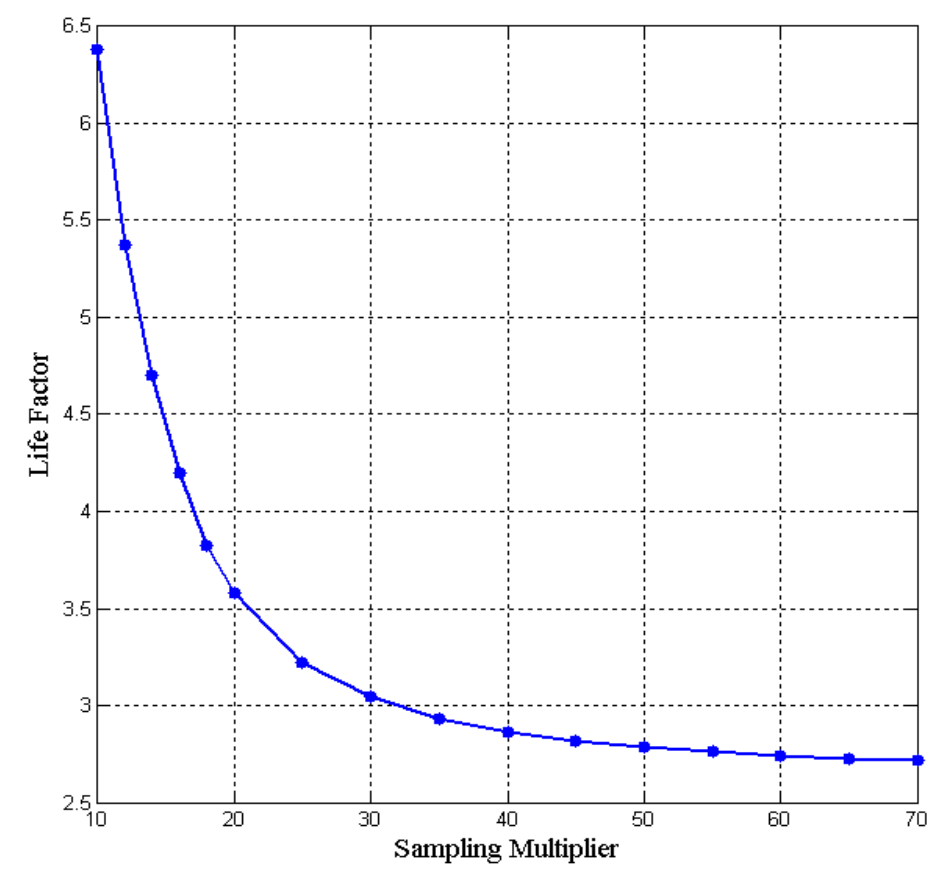

- Life factors calculated with sampling multiplier $=50$ are approximately $2.5 \%$ higher than those calculated using a sampling multiplier $=70$.

- Life factors reported previously may represent slight over-predictions. 


\section{Conclusions}

- Finite life predictions can be two or more times longer if dither is considered.

- Unreasonable to assume the consideration of dither always results in life predictions two or more times longer.

- Life factor results and sensitivity studies provide information to determine if the effects of dither are an important analysis consideration. 